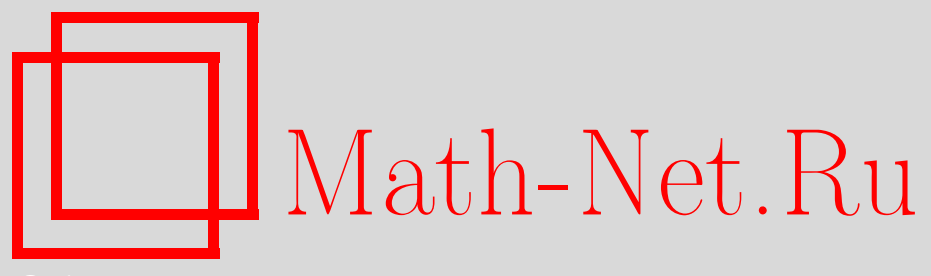

Ж. Бургейн, Б. С. Кашин, О равномерном приближении частной суммы ряда Дирихле более короткой суммой, Матем. заметки, 2010, том 87, выпуск 2, 309-310

DOI: https://doi.org/10.4213/mzm8593

Использование Общероссийского математического портала Math-Net.Ru подразумевает, что вы прочитали и согласны с пользовательским соглашением http://www . mathnet.ru/rus/agreement

Параметры загрузки:

IP : 52.23 .180 .231

26 апреля 2023 г., 13:29:45

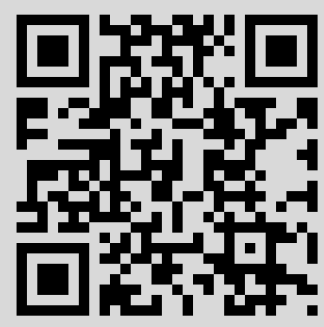




\section{О равномерном приближении частной суммы ряда Дирихле более короткой суммой}

Ж. Бургейн, Б. С. Кашин

1. Введение и формулировка основного результата. Установленная в этой заметке теорема по формулировке и методу доказательства может быть отнесена к группе результатов об оценках поперечников, полученных с использованием техники функционального анализа и теории вероятностей. При этом основной причиной для ее рассмотрения была некоторая аналогия с утверждениями, находящими важные приложения в теории чисел (см., например, [1; гл. 3]). Отметим также, что интерес к результатам общего характера о равномерном приближении частной суммы ряда Дирихле более короткой суммой высказывал несколько лет назад Карацуба.

Ниже мы используем обычные обозначения: $\mathbb{R}, \mathbb{Z}$ - множества действительных и целых чисел соответственно, $\mathbb{C}^{N}, N=1,2, \ldots,-N$-мерное комплексное пространство. Для $x=\left\{x_{i}\right\}_{i=1}^{N} \in \mathbb{C}^{N}, 1 \leqslant p<\infty$

$$
\|x\|_{L_{p}^{N}}=\left(\frac{1}{N} \sum_{i=1}^{N}\left|x_{i}\right|^{p}\right)^{1 / p}, \quad\|x\|_{L_{\infty}^{N}}=\max _{1 \leqslant i \leqslant N}\left|x_{i}\right| .
$$

Через $\operatorname{dist}_{E}(f, L)$ мы обозначаем расстояние от элемента $f$ до множества $L$ в нормированном пространстве $E ;|\Lambda|$ - число элементов в конечном множестве $\Lambda$. Наконец, $\operatorname{span}\left(\left\{\varphi_{i}\right\}_{i \in \Lambda}\right)$ - линейная оболочка системы элементов $\left\{\varphi_{i}\right\}_{i \in \Lambda}$ линейного пространства.

Tеорема. Пусть $T \in \mathbb{R}, T \geqslant 1, N \in \mathbb{Z}, N \geqslant 1, T>(\log N)^{10} u \rho \in(0,1)$. Hайдется подмножество и,ельх чисел

$$
\Lambda \subset \mathbb{Z} \cap\left[0, N\left(1+\frac{(\log N)^{7}}{T}\right)\right]
$$

такое, что $|\Lambda| \leqslant \rho \min \{N, T\}$ и для любого полинома $f(t)$ вида $f(t)=\sum_{n=0}^{N} a_{n} n^{i t} c$

$$
\sum_{n=0}^{N}\left|a_{n}\right|^{2} \leqslant 1, \quad \frac{1}{2 T} \int_{-T}^{T}|f(t)|^{2} d t \leqslant 1
$$

найдется полином $g(t)=\sum_{n \in \Lambda} b_{n} n^{i t} c$

$$
\sup _{|t| \leqslant T / 2}|g(t)-f(t)| \leqslant C[\log (N+T)]^{18} \rho^{-1 / 2}
$$

(здесь и ниже $C$ - абсолютная постоянная).

2. О доказательстве теоремы. Более тридцати лет назад в теории поперечников было установлено [2], что некоторые $k$-мерные подпространства $\mathbb{R}^{N}$ очень хорошо аппроксимируют в равномерной метрике евклидов шар в $\mathbb{R}^{N}$ даже при $N$ гораздо больших $k$. В связи с задачей о тригонометрических поперечниках, введенных в [3], естественно возникает вопрос о существовании таких примеров среди "координатных" подпространств, т.е. в данном случае подпространств, порожденных каким-то набором элементов дискретной тригонометрической системы. Первый результат о существовании "координатных"

Работа второго автора выполнена при поддержке Российского фонда фундаментальных исследований (гранты №№ 08-01-00598-a, 08-01-00799-а, 09-01-12173-офи_м).

(C) Ж. Бургейн, Б. С. КАшин, 2010 
тригонометрических подпространств в $\mathbb{R}^{N}$ размерности $k \leqslant \gamma N, \gamma<1$ - абсолютная постоянная, $N=2,3, \ldots$, хорошо приближающих в равномерной метрике евклидов шар был получен Бургейном и усовершенствован Талаграном [4]. При этом порядок приближения, доставляемого такими подпространствами, ухудшается лишь на логарифмический множитель по сравнению с подространствами общего вида. Недавно Гедоном, Мендельсоном, Пажором и Томчак-Ягерманн [5] был получен результат общего характера о существовании "координатных" подпространств малой размерности с хорошими аппроксимационными свойствами, порожденных элементами произвольного равномерно ограниченного ортонормированного базиса в $L_{2}^{N}$. Схема доказательства, использованная в [5], применяется и при доказательстве следующего результата.

ПредложениЕ. Пусть задан набор элементов $\left\{\varphi_{i}\right\}_{i=1}^{n} \subset L_{2}^{N} c\left\|\varphi_{i}\right\|_{L_{\infty}^{N}} \leqslant K, i=$ $1,2, \ldots, n$. Для любого $m, 1 \leqslant m \leqslant n$, найдется подмножество $\Lambda \subset\{1, \ldots, n\}$ такое, чmo $|\Lambda|=m u$

$$
\sup _{\left\{a_{i}\right\}_{i=1}^{n}, \sum\left|a_{i}\right|^{2} \leqslant 1} \operatorname{dist}_{L_{\infty}^{N}}\left(\sum_{i=1}^{n} a_{i} \varphi_{i}, \operatorname{span}\left(\varphi_{i}, i \in \Lambda\right)\right) \leqslant C \cdot K(\log N)^{7 / 2} \sqrt{\frac{n}{m}} .
$$

Отметим, что в приведенном предложении, доказательство которого использует также теорему 6 из [6], отсутствует требование ортогональности системы $\left\{\varphi_{i}\right\}$.

В доказательстве теоремы помимо методов геометрии банаховых пространств используется и техника гармонического анализа.

\section{СПИСОК ЦИТИРОВАННОЙ ЛИТЕРАТУРЫ}

[1] С. М. Воронин, А.А. Карацуба, Дзета-функция Римана, Физматлит, М., 1994. [2] Б. С. Кашин, Изв. АН СССР. Сер. матем., 41:2 (1977), 334-351. [3] Р. С. Исмагилов, УМH, 29:3 (1974), 161-178. [4] M. Talagrand, Israel J. Math., 108 (1998), 173-191. [5] O. Guédon, S. Mendelson, A. Pajor, N. Tomczak-Jaegermann, Positivity, 11:2 (2007), 269-283. [6] J. Bourgain, A. Pajor, S. J. Szarek, N. Tomczak-Jaegermann, Geometric Aspects of Functional Analysis, Lecture Notes in Math., 1376, Springer-Verlag, Berlin, 1989, $50-63$.

\section{Ж. Бургейн}

Поступило

Institute for Advanced Study, 30.10 .2009

School of Mathematics, Princeton

E-mail: bourgain@math.ias.edu

\section{Б. С. Кашин}

Математический институт им. В. А. Стеклова РАН

E-mail: kashin@mi.ras.ru 